\title{
ALGUMAS OBSERVAÇO์ES SOBRE O CICLO REPRODUTIVO ANUAL DE FÊMEAS DO GAMBÁ DIDELPHIS ALBIVENTRIS (LUND, 1841) (MARSUPIALIA, DIDELPHIDAE) EM POPULAÇOEES NATURAIS NO ESTADO DE MINAS GERAIS, BRASIL ${ }^{1}$
}

\author{
Sônia Elias Rigueira ${ }^{2}$ \\ Célio Murilo de Carvalho Valle ${ }^{2}$ \\ José Benedito Malta Varejão ${ }^{3}$ \\ Patrícia Velloso de Albuquerque ${ }^{2}$ \\ José Carlos Nogueira ${ }^{4}$
}

\begin{abstract}
Females of white-eared opossum Didelphis albiventris (Lund, 1841) from natural population of the "cerrado", State of Minas Gerais, Brazil, present a seasonal reproductive activity. Young-pouches were found in females captured from August to March. During the months of April to July youngs were not present in the pouch. The only two females captured in July showed pregnant uterus. All females collected in August and September were litter positive. The average number of individuals per litter was $7.04 \pm 1.9$.
\end{abstract}

\section{INTRODUÇÃO}

Poucas são as informaçбes relativas à história natural das espécies de Didelphis no Brasil (Miranda-Ribeiro, 1936; Davis, 1945; Valle et al. 1981; Streilein, 1981; Streilein, $1982 a$ e $b$; Fonseca et al., 1982), apesar de muitas pesquisas laboratoriais serem desenvolvidas sobre estes animais (Rocha-Miranda, 1978). 0 mesmo poderíamos salientar com relação a trabalhos de taxonomia e zoogeografia (Hershkovitz, 1969; Cerqueira, 1982; Varejão \& Valle, 1982).

O objetivo do presente trabalho é registrar algumas observaçбes sobre o ciclo reprodutivo anual de fêmeas do gambá Didelphis albiventris, baseado na presença ou não de filhotes na bolsa.

1 Financiado pelo CPq/UFMG e FINEP.

2 Departamento de Zoologia do ICB/UFMG, Belo Horizonte, MG.

3 Departamento de Patologia, Setor Parasitologia - UFES, Vitória, ES.

4 Departamento de Morfologia do ICB/UFMG, Belo Horizonte, MG. Pesquisador do CNPq. 


\section{MATERIAL E MÉTODOS}

Aleatoriamente, durante os anos de 1978, 1979, 1981, 1982, 1984 e 1985 foram capturados 104 gambás de orelha branca (36 machos e 68 fêmeas) em regiáo de cerrado no Estado de Minas Gerais (Fig. 1), Brasil. Os animais foram capturados em armadilhas com isca de banana. No laboratório, depois de anestesiados e sacrificados, os filhotes foram retirados da bolsa, fixados em formol a $10 \%$ e conservados em álcool a $70 \%$, exceto as fêmeas capturadas em Belo Horizonte (MG), no período de janeiro a março e dezembro de 1984 e de janeiro a março de 1985, em cujos animais, após a anestesia se procedeu somente a contagem dos filhotes na bolsa. Cada ninhada recebeu o mesmo número da mãe que, conforme o caso, foi taxidermizada. Este material se encontra registrado e depositado na coleção do Departamento de Zoologia do Instituto de Ciências Biológicas da Universidade Federal de Minas Gerais, Belo Horizonte. As medidas dos filhotes foram feitas em animais fixados e se encontram na Fig. 3. Detalhes sobre as técnicas de captura podem ser encontradas em Varejão \& Valle, 1982.

Algumas observaçбes complementares foram tomadas das fichas de protocolos de necrópsia nos Laboratórios de Neuroanatomia e Reprodução Animal do Departamento de Morfologia do ICB/UFMG.

\section{RESULTADOS}

Os municípios de Minas Gerais, Brasil, onde foram feitas as capturas das fêmeas de Didelphis albiventris estão contidos no mapa representado pela Fig. 1.

Conforme se verifica nas Figs. 2, 3 e 4, foram registradas fêmeas, em populaçбes naturais, com filhotes na bolsa nos meses de agosto a março, salientando-se que nos meses de fevereiro e março as fêmeas com filhotes na bolsa foram encontradas em menor freqüência. Todas as fêmeas capturadas em agosto e setembro possuíam filhotes na bolsa. Nos meses de abril a junho todas as fêmeas capturadas não apresentavam filhotes na bolsa; entretanto, as duas fêmeas capturadas em julho apresentavam úteros grávidos. $O$ número médio de filhotes (Figs. 2 e 3 ) na bolsa foi de $7,04 \pm 1,9$, variando de 02 a 10 filhotes.

\section{DISCUSSĀO}

Estudando os aspectos histológicos do sistema genital feminino de Didelphis albiventris em Minas Gerais, Mascarenhas (1980) registra fêmeas em estro nos meses de julho, outubro e novembro, e em anestro de fevereiro a junho. Tais informaçōes baseadas em 20 fêmeas capturadas confirmam 


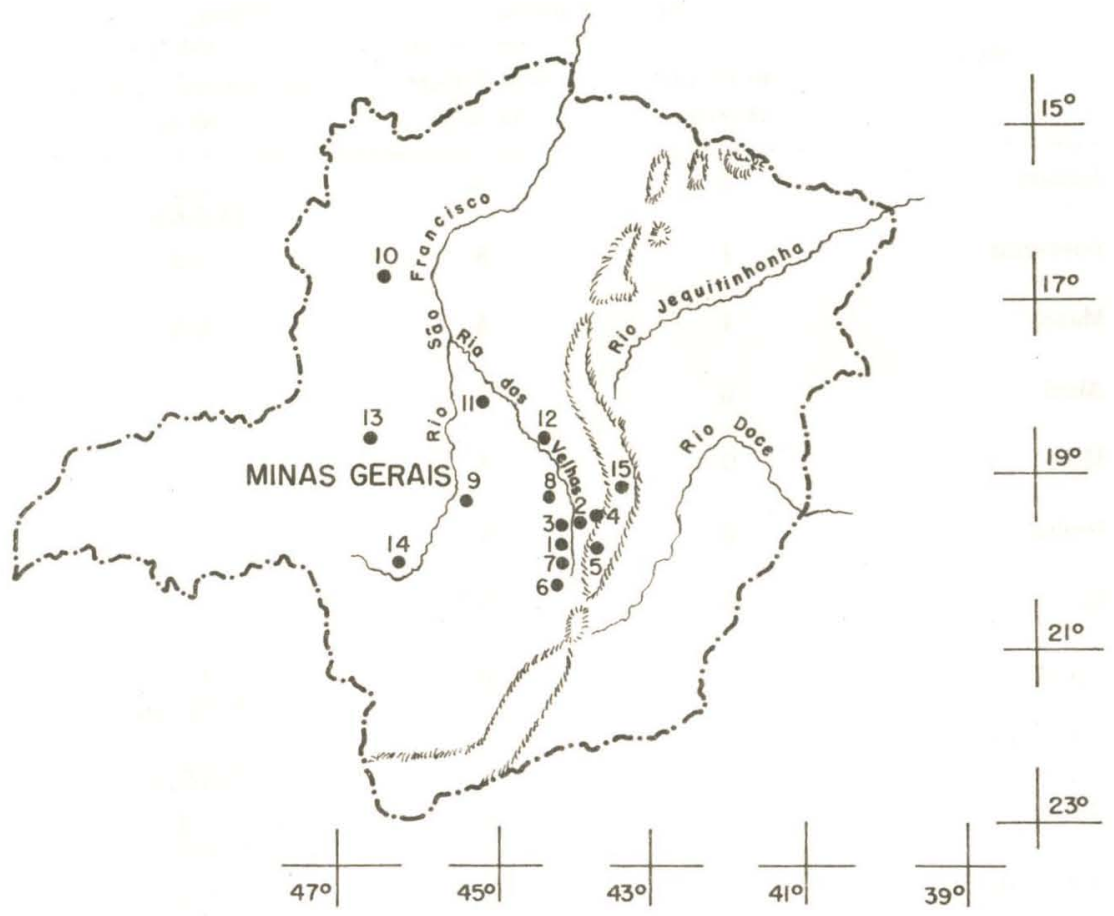

Fig. 1 - Mapa da distribuição dos municípios de Minas Gerais, Brasil, onde foram capturadas as fêmeas de Didelphis albiventris.

No Localidade .

01 Belo Horizonte

02 Santa Luzia

u3 Lagoa Santa

04 Pedro Leopoldo

05 Caeté

06 Glaura

$07 \quad$ Nova Lima

08 Sete Lagoas

09 Pompeu

10 Bonfinópolis de Minas

11 Lassance

12 Presidente Juscelino

13 Patos de Minas

14 Bambuí

15 São Gonçalo do Rio Abaixo

\begin{tabular}{|c|c|}
\hline & \\
\hline & $40^{\circ} w$ \\
\hline${ }^{\circ} 50$ 's & $43^{\circ} 40^{\prime}$ \\
\hline & \\
\hline & \\
\hline 's & $43^{\circ}$ \\
\hline & \\
\hline & $43^{\circ} 55 \mathrm{~T}$ \\
\hline${ }^{\circ} 29$ 's & $44^{\circ}$ \\
\hline & \\
\hline & $46^{\circ}$ \\
\hline${ }^{\circ} 58$ & $44^{\circ} 05^{\prime}$ \\
\hline & \\
\hline & \\
\hline & \\
\hline & \\
\hline
\end{tabular}


Fig. 2 - Número total de fêmeas de Didelphis albìventris capturadas mensalmente e número médio de filhotes por ninhada.

\begin{tabular}{|c|c|c|c|}
\hline \multirow{2}{*}{ Meses } & \multicolumn{2}{|c|}{ Número de fêmeas } & \multirow{2}{*}{$\begin{array}{c}\text { Número médio } \\
\text { de filhotes } \\
\text { por ninhada na bolsa } \\
\text { e variação }\end{array}$} \\
\hline & $\begin{array}{l}\text { Com filhotes } \\
\text { na bolsa }\end{array}$ & $\begin{array}{l}\text { Sem filhotes } \\
\text { na bolsa }\end{array}$ & \\
\hline Janeiro & 5 & 3 & $\begin{array}{c}6,2 \\
(4,0-8,0)\end{array}$ \\
\hline Fevereiro & 1 & 6 & 6,0 \\
\hline Março & 1 & 5 & 6,0 \\
\hline Abril & 0 & 3 & - \\
\hline Maio & 0 & 3 & - \\
\hline Junho & 0 & 7 & - \\
\hline Julho & 0 & $2 *$ & - \\
\hline Agosto & 5 & 0 & $\begin{array}{c}9,2 \\
(8,0-10,0)\end{array}$ \\
\hline Setembro & 7 & 0 & $\begin{array}{c}7,2 \\
(6,0-9,0)\end{array}$ \\
\hline Outubro & 2 & 5 & $\begin{array}{c}6,0 \\
(5,0-7,0)\end{array}$ \\
\hline Novembro & 2 & 3 & $\begin{array}{c}4,5 \\
(2,0-7,0)\end{array}$ \\
\hline Dezembro & 2 & 6 & $\begin{array}{c}8,5 \\
(8,0-9,0)\end{array}$ \\
\hline
\end{tabular}

* Fêmeas com útero grávido.

nossos achados relativos à presença de filhotes na bolsa nos meses por nós encontrados (agosto a março). Por outro lado, a deteç̧ão de anestro de fevereiro a junho também coincide com nossos resultados onde encontramos a grande maioria de fêmeas sem filhotes capturadas nesse período, salientando que os poucos filhotes encontrados em fevereiro e março foram fecundados em período de estro.

Consultando as fichas de protocolos de autópsias de D. albiventris do Departamento de Morfologia do ICB/UFMG nos anos de 1963, 1964, 1977, 1983 e 1984 encontram-se registros de fêmeas com filhotes na bolsa apenas nos meses de julho, agosto, outubro e janeiro. Das duas fêmeas autopsiadas em julho, uma (24-07-74) possuía filhotes na bolsa e a outra (24-07-64) possuía "embriøes" no útero. Pelos nossos resultados encontramos duas fêmeas capturadas em julho com úteros grávidos, sendo capturadas em 16 e 26-0779 , respectivamente. Embora não tenhamos encontrados fêmeas com filhotes na bolsa no mês de julho, isto pode ocorrer se se considerar que a gestação 
Fig. 3 - Relação das fêmeas de Didelphis albiventris capturadas com filhotes na bolsa no período de 1978 e 1979, em Minas Gerais, Brasil.

\begin{tabular}{|c|c|c|c|c|c|c|c|c|}
\hline \multirow{2}{*}{$\begin{array}{l}\text { No de coleção } \\
\text { do Departamento } \\
\text { de Zoologia }\end{array}$} & \multirow{2}{*}{ Procedência } & \multirow{2}{*}{$\begin{array}{l}\text { Data de } \\
\text { Captura }\end{array}$} & \multirow{2}{*}{$\begin{array}{l}\text { Comprimento } \\
\text { total da } \\
\text { fêmea }(\mathrm{mm})\end{array}$} & \multirow{2}{*}{$\begin{array}{l}\text { No de } \\
\text { filhotes }\end{array}$} & \multicolumn{4}{|c|}{ Medidas de comprimento dos filhotes (mm) } \\
\hline & & & & & Cabeça & Corpo & Cauda & Total \\
\hline- & Belo Horizonte & $25-09-78$ & - & 6 & $\begin{array}{c}32,9 \\
(31,0-34,0)\end{array}$ & $\begin{array}{c}68,1 \\
(60,0-74,8)\end{array}$ & $\begin{array}{c}69,0 \\
66,1-69,6)\end{array}$ & $\begin{array}{c}169,4 \\
159,7-178,2)\end{array}$ \\
\hline 529 & Santa Luzia & $01-11-78$ & - & 2 & $\begin{array}{c}51,2 \\
(50,9-51,5)\end{array}$ & $\begin{array}{c}105,1 \\
(103,0-107,3)\end{array}$ & 156,6 & 315,4 \\
\hline 640 & Pres. Juscelino & $16-07-79$ & $656^{*}$ & & & & & \\
\hline 639 & Pres. Juscelino & $26-07-79$ & $667 *$ & & & & & \\
\hline 648 & Lassance & $17-08-79$ & 652 & 9 & $\begin{array}{c}8,9 \\
(7,8-9,7)\end{array}$ & $\begin{array}{c}18,5 \\
(17,7-19,3)\end{array}$ & $\begin{array}{c}6,3 \\
5,5-7,2)\end{array}$ & $\begin{array}{c}33,8 \\
31,4-36,2)\end{array}$ \\
\hline 647 & Lassance & $17-08-79$ & 602 & 9 & $\begin{array}{c}11,8 \\
(11,4-12,4)\end{array}$ & $22,5-25,5)$ & $\begin{array}{c}10,6 \\
9,3-11,5)\end{array}$ & $\begin{array}{c}46,7 \\
(45,0-48,1)\end{array}$ \\
\hline 654 & Bonfinópolis de Minas & $17-08-79$ & 687 & 10 & $\begin{array}{c}11,0 \\
(10,5-11,4)\end{array}$ & $\begin{array}{c}22,5 \\
(20,5-23,7)\end{array}$ & $(9,3-11,0)$ & $\begin{array}{c}44,3 \\
(41,2-45,8)\end{array}$ \\
\hline 649 & Lassance & $17-08-79$ & 650 & 10 & $\begin{array}{c}13,9 \\
(13,0-14,8)\end{array}$ & $\begin{array}{c}31,3 \\
(27,7-33,6)\end{array}$ & $(12,2-14,9)$ & $\begin{array}{c}59,2 \\
(53,9-64,2)\end{array}$ \\
\hline 656 & S. Gonçalo do Rio Abaixo & $31-08-79$ & 682 & 8 & $\begin{array}{c}19,1 \\
(17,6-19,8)\end{array}$ & $\begin{array}{c}39,7 \\
(38,0-46,0)\end{array}$ & $\begin{array}{c}22,7 \\
(18,2-25,5)\end{array}$ & $\begin{array}{c}81,5 \\
(74,6-90,4)\end{array}$ \\
\hline 650 & Belo Horizonte & 03-09-79 & 698 & 7 & $\begin{array}{c}13,5 \\
(13,0-15,3)\end{array}$ & $\begin{array}{c}26,6 \\
(25,0-28,8)\end{array}$ & $\begin{array}{c}14,3 \\
(12,2-14,9)\end{array}$ & $\begin{array}{c}54,5 \\
(50,3-56,7)\end{array}$ \\
\hline 652 & Belo Horizonte & $03-09-79$ & 646 & 9 & $\begin{array}{c}16,0 \\
(15,3-16,7)\end{array}$ & $\begin{array}{c}40,4 \\
(38,9-45,3)\end{array}$ & $\begin{array}{c}17,2 \\
(16,8-18,2)\end{array}$ & $\begin{array}{c}73,6 \\
(71,6-78,6)\end{array}$ \\
\hline 661 & S. Gonçalo do Rio Abaixo & $06-09-79$ & 665 & 6 & $\begin{array}{c}21,7 \\
(20,6-22,8)\end{array}$ & $\begin{array}{c}41,2 \\
(39,1-46,0)\end{array}$ & $\begin{array}{c}28,6 \\
(25,5-30,2)\end{array}$ & $\begin{array}{c}91,5 \\
(87,7-98,4)\end{array}$ \\
\hline
\end{tabular}

* Fêmeas com gravidez uterina.

(Continua) 
Continuação da Fig. 3

\begin{tabular}{|c|c|c|c|c|c|c|c|c|}
\hline \multirow{2}{*}{$\begin{array}{l}\text { Nọ de coleçã̀ } \\
\text { do Departamento } \\
\text { de Zoologia }\end{array}$} & \multirow{2}{*}{ Procedência } & \multirow{2}{*}{$\begin{array}{l}\text { Data de } \\
\text { Captura }\end{array}$} & \multirow{2}{*}{$\begin{array}{l}\text { Comprimento } \\
\text { total da } \\
\text { fêmea ( } \mathrm{mm} \text { ) }\end{array}$} & \multirow{2}{*}{$\begin{array}{l}\text { No de } \\
\text { filhotes }\end{array}$} & \multicolumn{4}{|c|}{ Medidas de comprimento dos filhotes (mm) } \\
\hline & & & & & Cabeça & Corpo & Cauda & Total \\
\hline 689 & S. Gonçalo do Rio Abaixo & $18-09-79$ & 620 & 7 & $\begin{array}{c}17,8 \\
(17,4-18,6)\end{array}$ & $\begin{array}{c}34,5 \\
(33,4-46,4)\end{array}$ & $\begin{array}{c}25,9 \\
(23,0-29,0)\end{array}$ & $\begin{array}{c}78,3 \\
(75,4-81,5)\end{array}$ \\
\hline 678 & S. Gonçalo do Rio Abaixo & $25-09-79$ & 715 & 8 & $\begin{array}{c}26,0 \\
(24,0-27,0)\end{array}$ & $\begin{array}{c}48,5 \\
(48,0-52,0)\end{array}$ & $\begin{array}{c}42,8 \\
(39,4-51,1)\end{array}$ & $\begin{array}{c}117,4 \\
(109,3-131,1)\end{array}$ \\
\hline 680 & S. Gonçalo do Rio Abaixo & $26-09-79$ & 645 & 9 & $\begin{array}{c}26,8 \\
(26,0-27,9)\end{array}$ & $\begin{array}{c}54,8 \\
(51,5-65,5)\end{array}$ & $\begin{array}{c}49,3 \\
(49,7-52,2)\end{array}$ & $\begin{array}{c}131,0 \\
(124,8-144,7)\end{array}$ \\
\hline 703 & Pedro Leopoldo & $17-10-79$ & 650 & 5 & $\begin{array}{c}39,5 \\
(39,3-40,0)\end{array}$ & $\begin{array}{c}84,1 \\
(81,5-87,9)\end{array}$ & $\begin{array}{c}94,6 \\
(93,9-98,6)\end{array}$ & $\begin{array}{c}218,2 \\
(208,0-226,3)\end{array}$ \\
\hline 707 & Pedro Leopoldo & $18-10-79$ & 691 & 7 & $\begin{array}{c}41,2 \\
(40,0-43,6)\end{array}$ & $\begin{array}{c}85,8 \\
(79,9-91,0)\end{array}$ & $\begin{array}{c}100,4 \\
(97,4-100,9)\end{array}$ & $\begin{array}{c}227,3 \\
(223,0-232,4)\end{array}$ \\
\hline 710 & Bambuí & $07-11-79$ & 669 & 7 & $\begin{array}{c}26,2 \\
(24,5-27,0)\end{array}$ & $\begin{array}{c}47,2 \\
(40,5-52,4)\end{array}$ & $\begin{array}{c}41,1 \\
(37,1-46,4)\end{array}$ & $\begin{array}{c}114,6 \\
(103,2-119,4)\end{array}$ \\
\hline
\end{tabular}


uterina de $D$. albiventris é de 13 dias (Talice \& Lagomarsino, 1959); portanto, nas fêmeas em que o estro se inicia na $1^{\text {a }}$ metade de julho, a ocorrência de filhotes na bolsa é um achado normal, o que ocorreria com a fêmea capturada e sacrificada em 16-07-79.

Descrevendo a biometria do sistema genital masculino de $D$. albiventris durante o ciclo reprodutivo anual, Nogueira (1985) salienta que o peso da próstata e das glândulas bulbo-uretrais (Cowper) laterais e intermédias apresentam acentuado aumento ponderal de junho a janeiro, período de reprodução. Como as fêmeas somente apresentam estro a partir de julho, o período de acasalamento seria entre julho e janeiro. $\mathbf{O}$ achado de fêmeas com filhotes na bolsa nos meses de fevereiro e março, em menor número, é decorrente de fecundaçбes ocorridas nos meses de novembro, dezembro ou janeiro. A única fêmea capturada em março (20-03-84) apresentava 06 filhotes com $\pm 9,0 \mathrm{~cm}$ de comprimento (crown-rump), tamanho médio de filhotes prestes a saírem da bolsa.

Hill (1918), capturando fêmea de $D$. marsupialis entre julho e outubro no Estado do Rio de Janeiro, encontrou fêmeas grávidas e fêmeas com filhotes na bolsa durante o mês de julho. Em agosto, setembro e outubro ele somente encontrou fêmeas com filhotes na bolsa. Tais achados, embora para $D$. marsupialis, são semelhantes aos por nós encontrados em $D$. albiventris, em Minas Gerais.

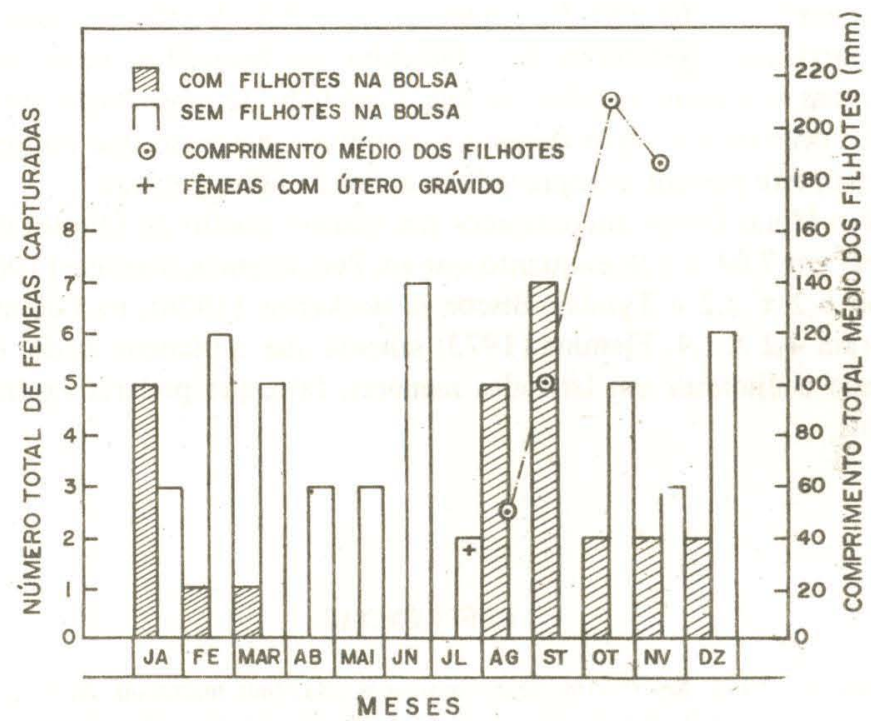

Fig. 4 - Número total de fêmeas capturadas mensalmente e comprimento total mếdio dos filhotes na bolsa de Didelphis albiventris, capturados nos anos de 1978 a 1982, 1984 e 1985. 
Por outro lado, Davis (1945), capturando fêmeas de D. marsupialis no Estado do Rio de Janeiro, no período de agosto de 1942 a setembro de 1943, registrou fêmeas com filhotes na bolsa de agosto a janeiro, confirmando os achados de Hill (1918). Nossos resultados são semelhantes aos encontrados por esses autores e demonstram haver um sincronismo na atividade reprodutiva das duas espécies de Didelphis nos Estados do Rio de Janeiro e Minas Gerais.

Varejão \& Valle (1982) demonstraram que D. marsupialis em Minas Gerais e Espírito Santo apresenta um período de reprodução ativo compreendido entre julho e março, semelhante ao por nós encontrado para $D$. albiventris, em Minas Gerais.

Este padrão de atividade reprodutiva em Didelphis encontrado nos diferentes estados do Brasil (Minas Gerais, Rio de Janeiro e Espírito Santo) é diferente do descrito por Cerqueira (1984), em $D$. albiventris, estudado em três diferentes regiōes do nordeste brasileiro, onde salienta que o início da estação de reprodução estaria relacionado com a quantidade de precipitação pluviométrica.

O ciclo reprodutivo de $D$. albiventris na Colômbia foi estudado por Tyndale-Biscoe \& Mackenzie (1976) que demonstraram uma maior atividade reprodutiva de março a setembro. Telford et al. (1979), estudando $D$. marsupialis na Venezuela, determinaram o período de reprodução de fevereiro a outubro, período também encontrado por Fleming (1973) para D. marsupialis na zona do Canal do Panamá e por Hunsaker (1977) e Tyndale-Biscoe \& Mackenzie (1976) para D. virginiana nos USA. Verifica-se, deste modo, que a atividade reprodutiva dos Didelphis do hemisfério norte estudados apresentam o mesmo padrão, ou seja, o período de reprodução abrangendo inverno, primavera e verão (janeiro a setembro), ao passo que no hemisfério sul, Brasil, este período compreende os meses de julho a março.

Em Minas Gerais encontramos um número médio de filhotes de $D$. albiventris em $7.04 \pm 1,9$, enquanto que em Pernambuco, Streilein $(1982 b)$ encontrou 6,2 $\pm 2,2$ e Tyndale-Biscoe \& Mackenzie (1976), na Colômbia, encontraram 4,2 $\pm 1,4$. Fleming (1973) salienta que o número médio de filhotes tende a diminuir em latitudes menores, fato que poderia explicar estes achados.

\section{REFERENCIAS}

Cerqueira, R., 1982. South American landscapes and their mammals. In Mares, M.A. \& Genoways, H.H. Mammalian Biology in South America. The Pymatuning Symposia in Ecology. University of Pittsburg.6:53-75.

Cerqueira, R., 1984. Reproduction de Didelphis albiventris dans le nord-est du Brésil. (Polyprotodontia, Didelphidae). Mammalia 48:95-104.

Dacis, D.E., 1945. The annual cycle of plants, mosquitoes, birds, and mammals in two Brazilian forests. Ecol. Mon. $15: 244-295$. 
Fleming, T.H., 1973. The reproductive cycle of opossums and other mammals in the Panama canal zone. J. Mammal. 54:439-455.

Fonseca. G.A.B., Redford, K.H. \& Pereira, L.A., 1982. Notes on Didelphis albiventris (Lund, 1841) of central Brazil. Cienc. Cult. 34 :1359-1362.

Hershkovitz, P., 1969. The evolution of mammals on southern continents. VI. The recent mammals of the neotropical region: a zoogeographic and ecological review. Quart. Rev. Biol 44 : 1-70.

Hill, J.P., 1918. Some observations on the early development of Didelphis aurita. Quart. J. Microsc. Sci. 63:91-139.

Hunsacker, D., 1977. The biology of marsupials. Academic Press, New York.

Mascarenhas, A.E.A.S., 1980. Histologia e invervação do sistema genital feminino na fase de anestro no gambd Didelphis albiventris (Lund, 1841) - Didelphidae Marsupialia. Tese de Mestrado, Depto. de Morfologia, ICB/UFMG, Belo Horizonte, MG.

Nogueira, J.C., 1985. Biometria do sistema genital masculino do gambá Didelphis alliventris (Lund, 1841) durante o ciclo reprodutivo anual. Em preparação.

Rocha-Miranda, C.E. \& Lent, R., 1978. Opossum neurobiology. Acad. Bras. Cienc., Rio de Janeiro, 293 pp.

Streilein. K.E., 1981. Behavior, ecology, and distribution of South American marsupials. In Mares, M.A. \& Genoways, H.H. Mammalian Biology in South America. The Pymatuning Symposia in Ecology. University of Pittsburg 6 : 231-250.

Streilein, K.E., 1982a. Ecology of small mammals in semiarid Brasilian caatinga. I. Climate and faunal composition. Annals Carnegie Museum 51 : 79-107.

Streilein, K.E., 1982b. Ecology of small mammals in semiarid Brasilian caatinga. III. Reproductive biology and population ecology. Annals Carnegie Museum 51 : 251269.

Talice, R.V. \& Lagomarsino, J.C., 1959. Comportamiento sexual e nacimientos en cautividad de la "comadreja overa": Didelphis azarae. Congresso Sudamericano de Zoologia, 5(6) : 81-96.

Telford Jr., S.R., Gonzalez, J. \& Tonn, R.J., 1979. Reproducción y crecimiento de Didelphis marsupialis, un huésped reservorio primario de la enfermedad de Chagas em los llanos altos da Venezuela. Bol. Bir. Malariol. San, amb. XIX:44-56.

Tyndale-Biscoe, C.H. \& Mackenzie, R.B., 1976. Reproduction in Didelphis marsupialis and D. albiventris in Colombia. J. Mammal. $52: 249-265$.

Valle, C.M.C., Varejão, J.B.M., Rigueira, S.E. \& Albuquerque, P.V., 1981. Contribuição ao conhecimento do ciclo reprodutivo anual em populaçōes naturais de Didelphis marsupialis Linnaeus, 1758 e D. albiventris (Lund, 1841) em Minas Gerais. Res. VIII Cong. Bras. Zool., Brasília, DF. 130-131.

Varejão, J.B.M. \& Valle, C.M.C., 1982. Contribuição ao estudo da distribuição geográfica das espécies do gênero Didelphis (Mammalia-Marsupialia) no Estado de Minas Gerais, Brasil. Lundiana $2:$ 5-55. 